\title{
Enhanced second-harmonic generation of asymmetric Au@CdSe heterorods
}

\author{
Biao $\mathrm{Wu}^{1,2 \dagger}$, Peng-Fei Wang ${ }^{1 \dagger}$, Yun-Hang Qiu ${ }^{1 \dagger}$, Shan Liang ${ }^{3}$, Zhi-Yong Wu ${ }^{1}$, Li Zhou ${ }^{1^{*}}$ and \\ Qu-Quan Wang ${ }^{1,4^{*}}$
}

\begin{abstract}
The plasmon-enhanced second-harmonic generation (SHG) of Au nanorods (NRs), Au-Ag NRs, and asymmetric Au@CdSe heterorods are comparatively investigated. The Au@CdSe tip- and side-heterorods are synthesized by precisely controlling the concentration of $\mathrm{Cd}^{2+}$ precursor, and the plasmon resonances can be well tuned simultaneously. The SHG of asymmetric Au@CdSe heterorods has been greatly enhanced with up to 617.7-fold enhancement compared with the Au NRs because of the asymmetric morphology and local field enhancement. Moreover, the harmonic energy transfer induced by the local interband transition of Au has also been demonstrated by the excitation-wavelengthdependent SHG in the Au NRs and Au@CdSe side-heterorods, while it is negligible in the Au-Ag NRs and Au@CdSe tipheterorods because of the presence of Ag. The plasmon-enhanced SHG of asymmetric Au@CdSe heterorods enables potential applications ranging from sensing to biological spectroscopy.
\end{abstract}

Keywords: second-harmonic generation, surface plasmon resonance, metal-semiconductor heterorods, asymmetric nanostructure

\section{INTRODUCTION}

Plasmon resonance of the metal nanostructures strongly enhances the local electromagnetic fields and various light-matter interactions [1-4], which has diverse applications ranging from optical information processing to photocatalysis and bioimaging [5-11]. The combination of plasmonics and nonlinear optics gives rise to a new research field called nonlinear plasmonics [12-14]. The nonlinear optical processes involving multi-photon excitations are much more sensitive to the enhanced local field compared with the linear processes. Various nonlinear optical processes, including second- and thirdharmonic generation (SHG and THG) [15-18], four-wave mixing [19,20], nonlinear Kerr effect [21,22], and multiphoton luminescence [23,24], have been observed in plasmonic nanostructures.

SHG is related to the second-order susceptibility $\chi^{(2)}$, whereby two photons of an incident laser at the fundamental frequency $\left(\omega_{\mathrm{FW}}\right)$ are absorbed simultaneously and then one photon at the second-harmonic frequency $\left(\omega_{\mathrm{SH}}\right.$ $\left.=2 \omega_{\mathrm{FW}}\right)$ is emitted. But SHG is forbidden $\left(\chi^{(2)}=0\right)$ in centrosymmetric materials within the electric dipole approximation [25-27]. The atomic nanostructures of the noble metals (such as $\mathrm{Au}$ and $\mathrm{Ag}$ ) common-used for plasmon resonance have centrosymmetry and therefore their SHG intensities are mainly contributed from the surface $[28,29]$. Great efforts have been made to enhance SHG of the plasmonic antennas [2,30,31]. Multipolar plasmon resonances [9,32], dual-resonant antennas $[2,30]$, and plasmonic Fano effect $[33,34]$ have been successfully used to enhance SH near field. Various noncentrosymmetric plasmonic nanostructures, such as lithographed L- [35], T- [36], U- [37], V- [30], and G- [38] shaped nanostructures as well as synthesized colloidal nanorods [39], nanostars [40], triangular nanoprisms [41], and nanocups [2], have been designed and fabricated to efficiently improve the far-field emission in-

\footnotetext{
${ }^{1}$ Key Laboratory of Artificial Micro- and Nano-structures of the Ministry of Education, Department of Physics, Wuhan University, Wuhan 430072, China

${ }^{2}$ Jiangxi Key Laboratory of Nanomaterials and Sensors, Provincial Key Laboratory of Optoelectronic and Telecommunication, College of Physics and Communication and Electronics, Jiangxi Normal University, Nanchang 330022, China

${ }^{3}$ Department of Physics, Hunan Normal University, Changsha 410081, China

${ }^{4}$ The Institute for Advanced Studies, Wuhan University, Wuhan 430072, China

$\dagger$ These authors contributed equally to this work.

* Corresponding authors (emails: qqwang@whu.edu.cn (Wang QQ); zhouli@whu.edu.cn (Zhou L))
} 
tensity of SHG.

In this study, we comparatively investigated the plasmon-enhanced SHG of Au nanorods (NRs), Au-Ag NRs, and Au@CdSe heterorods. The Au@CdSe heterorods were prepared through an asymmetric growth of CdSe nanocrystals on the Au-Ag NRs. By controlling the amount of $\mathrm{Cd}^{2+}\left(\mu_{\mathrm{Cd}}\right)$ for the CdSe growth, the morphology of Au@CdSe heterorods can be tuned from tipto side-heterorods precisely. Accordingly, the longitudinal surface plasmon resonance (L-SPR) of the heterorods shows gradual red-shift as the $\mu_{\mathrm{Cd}}$ gradually increases. Compared with the Au NRs and Au-Ag NRs, the SHG intensities of Au@CdSe heterorods have been greatly enhanced because of the asymmetric morphologies and local field enhancement. The harmonic energy transfer induced by the local interband transition of $\mathrm{Au}$ was observed through the excitation-wavelength-dependent SHG in the Au NRs and the Au@CdSe side-heterorods. The plasmon-enhanced SHG in the asymmetric Au@CdSe heterorods enables potential applications in sensing and biological spectroscopy.

\section{EXPERIMENTAL SECTION}

\section{Synthesis of Au@CdSe heterorods}

$\mathrm{Au}$ NRs were prepared by a commonly used seed-mediated growth method [42]. Subsequently, Au-Ag NRs were synthesized according to our previous report [43]. In a typical procedure, $15 \mathrm{~mL}$ of $\mathrm{Au}$ NRs was mixed with $15 \mathrm{~mL}$ of aqueous glycine acid solution $\left(200 \mathrm{mmol} \mathrm{L}^{-1}\right)$, and the $\mathrm{pH}$ value of mixture was adjusted to 6.8 by dropwise adding $2 \mathrm{~mol} \mathrm{~L}^{-1} \mathrm{NaOH}$. Then $50 \mu \mathrm{L}$ of $100 \mathrm{mmol} \mathrm{L}^{-1} \mathrm{AgNO}_{3}$ was added, and the well-mixed mixture was left undisturbed at $30^{\circ} \mathrm{C}$ for $20 \mathrm{~h}$. The solution color turned from brick-red into dark-red, indicating the formation of $\mathrm{Au}-\mathrm{Ag}$ NRs. The product was directly used as the seed for the synthesis of Au@CdSe heterorods.

Au@CdSe heterorods were synthesized by mixing $4 \mathrm{~mL}$ of the obtained $\mathrm{Au}-\mathrm{Ag} \mathrm{NRs}, 12 \mathrm{mg}$ of selenium powder, $80 \mu \mathrm{L}$ of $10 \mathrm{mmol} \mathrm{L}^{-1} \mathrm{NaBH}_{4}$, and a certain amount of $100 \mathrm{mmol} \mathrm{L}^{-1} \mathrm{Cd}\left(\mathrm{NO}_{3}\right)_{2}(25,50,75,100,125,200$ and $250 \mu \mathrm{L}$ ). Then the $\mathrm{pH}$ value of the mixture was set at 8.0 with dropwise addition of $2 \mathrm{~mol} \mathrm{~L}^{-1} \mathrm{NaOH}$ aqueous solution. The reaction was carried out with vigorous stirring at $90^{\circ} \mathrm{C}$ for $2 \mathrm{~h}$. The products were collected by centrifugation and then washed with water once.

\section{Characterizations}

The morphology of the sample was characterized using transmission electron microscopy (TEM) and high-resolution TEM (HRTEM) with a JEOL $2010 \mathrm{HT}$ and a JEOL 2010 FET, respectively. The absorption spectra were obtained using a UV-vis-NIR spectrophotometer (Cary 5000, Varian). Energy-dispersive X-ray spectroscopy (EDX) was performed using an EDX spectrometer incorporated in HRTEM.

\section{Nonlinear measurements}

The nonlinear measurements of SHG were performed by a self-built micro-PL optical system with the samples deposited on fused quartz substrates. The concentrations of Au NRs in all the samples for the SHG measurement were tuned to be same. The signal from substrate is negligible in comparison with those of the samples. The nonlinear emission spectra were recorded at room temperature. The ultrafast laser pulses were generated using a mode-locked Ti/sapphire laser (Mira 900, Coherent) with a repetition rate of $76 \mathrm{MHz}$ and a pulse width of $150 \mathrm{fs}$. The detection system was a spectrometer (Spectrapro 2500i, Acton) integrated with a cooled charge-coupled device camera (PyLoN:100B_eXcelon, Princeton).

\section{RESULTS}

\section{Nanostructures and plasmon resonance of the asymmetric Au@CdSe heterorods}

Fig. 1 shows the schematic preparation diagram and TEM images of the Au@CdSe heterorods. The synthesis method is a two-step strategy based on Au NRs, including a deposition of intermediate Ag layer and a subsequent growth of CdSe [43,44]. Au NRs with uniform morphology were firstly prepared by a seed growth method. As shown in Fig. 1a and e, the bare Au NRs have an average length of $62.8 \pm 2.2 \mathrm{~nm}$ and an average diameter of $19.3 \pm 1.2 \mathrm{~nm}$, and the corresponding aspect ratio is 3.3 \pm 0.3 . Fig. $1 \mathrm{~b}$ and $\mathrm{f}$ show TEM images of Au-Ag NRs with an average length of $63.1 \pm 2.1 \mathrm{~nm}$ and an average diameter of $19.6 \pm 1.3 \mathrm{~nm}$. The silver deposition can be easily observed because the NRs slightly transform into a dumbbell shape.Au@CdSe heterorods with different morphologies were then synthesized and the morphology was controlled by the amount of $\mathrm{Cd}\left(\mathrm{NO}_{3}\right)_{2}$ precursor $\left(\mu_{\mathrm{Cd}}\right)$. When $\mu_{\mathrm{Cd}}=50 \mu \mathrm{L}$, the Au@CdSe tip-heterorods are generated (Fig. 1c and g) and the CdSe grows on one corner of the Au NRs. When $\mu_{\mathrm{Cd}}=200 \mu \mathrm{L}$, the CdSe grows from the corner to the side of the NRs, forming the Au@CdSe side-heterorods (Fig. 1d and 1h).

There are two reaction processes in the second step for the growth of CdSe: 1) the selenation of Ag by the sele- 
a
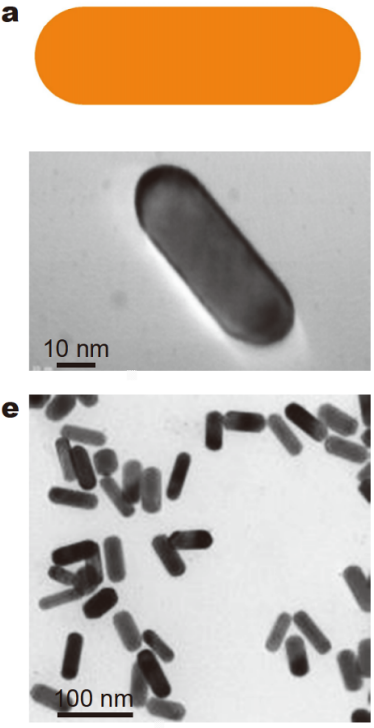

b
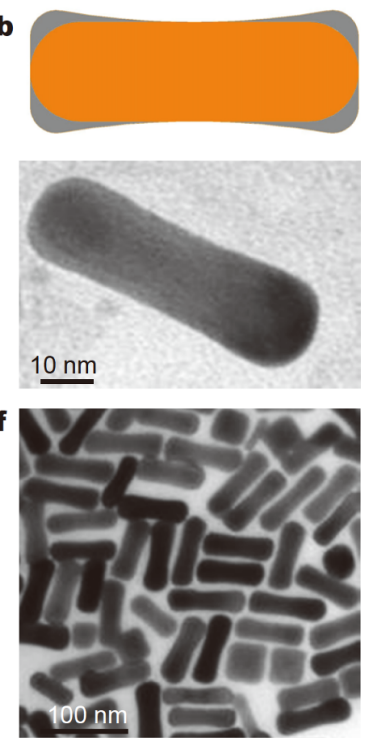
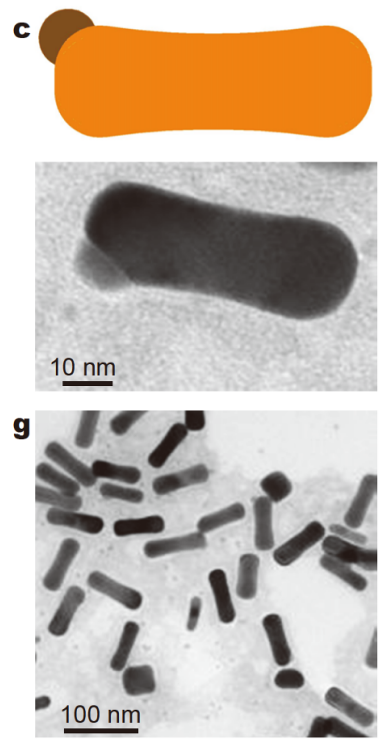

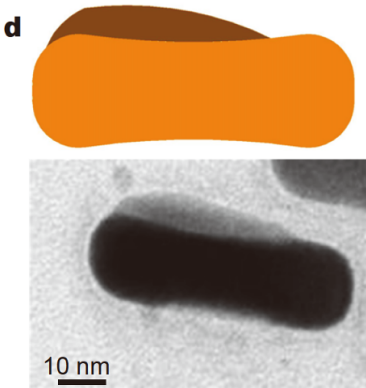

$10 \mathrm{~nm}$

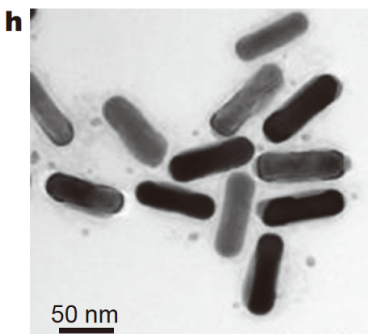

Figure 1 Illustrations and TEM images of different nanocrystals. (a, e) Au NRs, (b, f) Au-Ag NRs, (c, g) Au@CdSe tip-heterorods, and (d, h) Au@CdSe side-heterorods.

nium powder to form $\left.\mathrm{Ag}_{2} \mathrm{Se}\left(2 \mathrm{Ag}+\mathrm{Se} \rightarrow \mathrm{Ag}_{2} \mathrm{Se}\right) .2\right)$ The $\mathrm{Ag}_{2} \mathrm{Se}$ acts as seeds for the growth of $\mathrm{CdSe}\left(4 \mathrm{Cd}^{2+}+4 \mathrm{Se}+\right.$ $\left.\mathrm{BH}_{4}^{-}+3 \mathrm{H}_{2} \mathrm{O} \rightarrow 4 \mathrm{CdSe}+\mathrm{B}(\mathrm{OH})_{3}+7 \mathrm{H}^{+}\right)$. The intermediate layer of $\mathrm{Ag}$ is applied for the growth of CdSe to overcome the large lattice mismatch between $\mathrm{Au}$ and $\mathrm{CdSe}$, as the $\mathrm{Ag}$ is spontaneously selenized in the second step and the overgrowth of CdSe can be initiated on the active $\mathrm{Ag}_{2} \mathrm{Se}$ sites. As shown in Fig. $1 \mathrm{~b}$ and $\mathrm{f}$, the dumbbell shape of Au-Ag NRs indicates the Ag layers dominate the corner sites of NRs. Then it is reasonable that the CdSe overgrowth starts at these active corner sites to form the Au@CdSe tip-heterorods. Due to the large lattice mismatch between $\mathrm{Au}$ and CdSe, once CdSe is nucleated at these active sites, the subsequent growth of CdSe would continuously proceed at these sites. The growth and extension of CdSe from the corners to the side surfaces form the Au@CdSe side-heterorods. As seen in Fig. $1 \mathrm{~g}$ and $\mathrm{h}$, the CdSe has been grown onto almost all the Au NRs. The high yield of CdSe overgrowth is due to the crucial role of $\mathrm{Ag}$ intermediate layers. During the CdSe growth, there exists an etching effect on the $\mathrm{Au}$ NRs, leading to a flat Au-CdSe interface $[43,45]$.

The extinction spectra of plasmonic metal nanocrystals are strongly related to their morphologies and surrounding environments. As shown in Fig. 2a, the evolution of extinction spectra during the preparation of $\mathrm{Au} @ \mathrm{CdSe}$ was recorded. The L-SPR band of Au NRs appears at $826 \mathrm{~nm}$, and the transverse SPR (T-SPR) band appears at $515 \mathrm{~nm}$. After the Au-Ag NRs are formed, the
L-SPR blue-shifts to $798 \mathrm{~nm}$ and the T-SPR is almost unchanged. The blue-shift is caused by the negative dielectric constant of Ag and the slight morphology variation. As the Au@CdSe heterorods are formed and the morphology is evolved, the corresponding L-SPR band also shifts regularly. When $\mu_{\mathrm{Cd}}=50 \mu \mathrm{L}$, the Au@CdSe tipheterorods are generated with the L-SPR band slightly red-shifted to $802 \mathrm{~nm}$. Compared with the Au-Ag NRs, the full width at half maximum (FWHM) is obviously increased. In this case, a small amount of CdSe has little effect on the aspect ratio of NRs, while the plasmon damping is increased due to the interaction with CdSe. When $\mu_{\mathrm{Cd}}=200 \mu \mathrm{L}$, the Au@CdSe side-heterorods are formed and the L-SPR red-shifts to $862 \mathrm{~nm}$. In general, as shown in Fig. $2 \mathrm{~b}$ and Fig. S1, when the $\mu_{\mathrm{Cd}}$ gradually increases, the corresponding L-SPR of Au@CdSe heterorods is gradually red-shifted, with the gradually increased FWHM. The T-SPR also shows a very slight redshift. The SPR red-shift is mainly caused by the increased effective environmental dielectric constant due to the CdSe overgrowth. The increased FWHM of L-SPR band implies the increased plasmon damping due to the increased amount of CdSe on the Au NRs. For all the NR samples, the band at the high-energy side of T-SPR is attributed to the interband transition of $\mathrm{Au}$.

\section{SHG spectra of the Au NRs and Au-Ag NRs}

Fig. 3a and b present the SHG emission spectra of $\mathrm{Au}$ $\mathrm{NRs}$ and $\mathrm{Au}-\mathrm{Ag} \mathrm{NRs}$ as the fundamental excitation wa- 

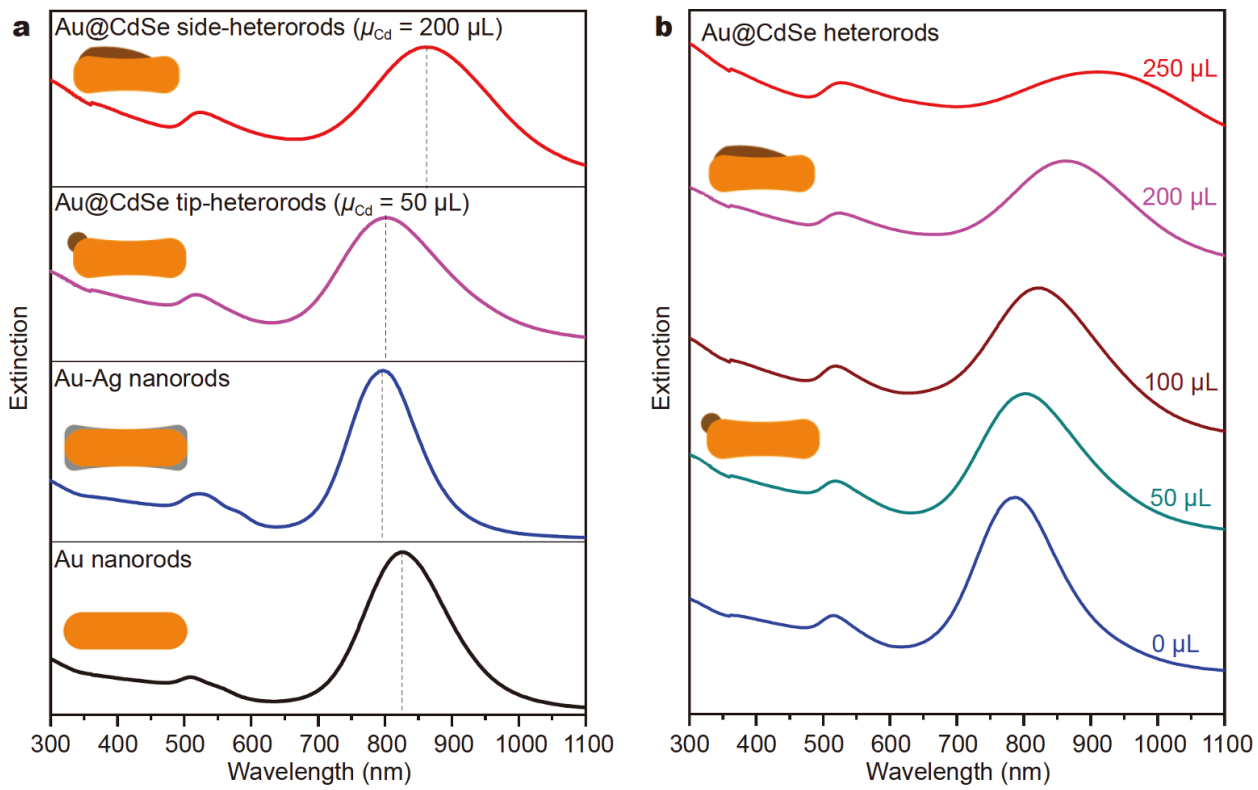

Figure 2 Extinction spectra of NRs in the preparation of Au@CdSe heterorods. (a) Extinction spectra of Au NRs, Au-Ag NRs and Au@CdSe heterorods with $\mu_{\mathrm{Cd}}=50$ and $200 \mu \mathrm{L}$. The L-SPR wavelength of the bare Au NRs is $826 \mathrm{~nm}$, and that of the Au-Ag NRs is $798 \mathrm{~nm}$. The L-SPR shifts to 802 and $862 \mathrm{~nm}$ when $\mu_{\mathrm{Cd}}=50$ and $200 \mu \mathrm{L}$, respectively. (b) Extinction spectra of Au@CdSe with different $\mu_{\mathrm{Cd}}$ at $0,50,100,200$, and $250 \mu \mathrm{L}$, respectively.

velength $\lambda_{\mathrm{FW}}$ is tuned from 750 to $930 \mathrm{~nm}$ with a step of $10 \mathrm{~nm}$. The emission spectra of Au NRs include an ultrasharp SHG peak and a background feature of two-photon luminescence (TPL), simultaneously. As for those of the $\mathrm{Au}-\mathrm{Ag}$ NRs, the SHG intensity is largely enhanced by the Ag deposition. The TPL background is less observed due to the largely enhanced SHG intensity as well as the Aginduced energy transfer and the red-shift of TPL emission [9]. The corresponding SHG intensity $I_{\mathrm{SHG}}(2 \omega)$ as a function of excitation wavelength of both the Au NRs and $\mathrm{Au}-\mathrm{Ag}$ NRs are shown in Fig. 3c. The excitation spectrum of Au NRs exhibits a main peak at $\lambda_{\mathrm{FW}}=860 \mathrm{~nm}$ and a weak satellite peak at $\lambda_{\mathrm{FW}}=830 \mathrm{~nm}$, which are near but not well matched with the L-SPR of Au NRs at $826 \mathrm{~nm}$. As the SHG emission wavelength from 375 to $465 \mathrm{~nm}$ (with the $\lambda_{\mathrm{FW}}$ ranging from 750 to $930 \mathrm{~nm}$ ) is located at the interband transition of $\mathrm{Au}$, the SHG is enhanced by the interband transition. The harmonic resonance energy transferring from the $\mathrm{SH}$ excitation to the interband linear excitation produces the two peaks in the excitation spectrum of $I_{\mathrm{SHG}}(2 \omega)$ [46]. The $I_{\mathrm{SHG}}(2 \omega)$ of $\mathrm{Au}-\mathrm{Ag}$ NRs shows a maximum of 48.8 -fold enhancement compared with that of the bare Au NRs, which is due to the large local field enhancement caused by the deposition of $\mathrm{Ag}$ [2]. However, the excitation spectrum of $\mathrm{Au}-\mathrm{Ag}$ NRs shows a maximum at $850 \mathrm{~nm}$ and the two-peak pattern is not obvious after the deposition of Ag.

\section{SHG spectra of the asymmetric Au@CdSe tip-heterorods}

We then investigated the SHG behaviors of the Au@CdSe heterorods with a small amount of CdSe deposition. The typical SHG emission spectra of Au@CdSe tip-heterorods with $\mu_{\mathrm{Cd}}=50 \mu \mathrm{L}$ are shown in Fig. $4 \mathrm{a}$. The corresponding $I_{\mathrm{SHG}}(2 \omega)$ as a function of $\lambda_{\mathrm{FW}}$ for the Au@CdSe heterorods with a small amount of CdSe deposition $\left(\mu_{\mathrm{Cd}}=25,50\right.$, and $75 \mu \mathrm{L}$ ) are presented in Fig. 4b. The excitation spectra for $I_{\mathrm{SHG}}(2 \omega)$ of all the Au@CdSe heterorods show a similar line-shape with one peak located at $\lambda_{\mathrm{FW}}=840 \mathrm{~nm}$. As the $\mu_{\mathrm{Cd}}$ increases, the $I_{\mathrm{SHG}}(2 \omega)$ firstly increases and then shows a decrease with $\mu_{\mathrm{Cd}}=75 \mu \mathrm{L}$. After the growth of CdSe, all the Au@CdSe heterorods have enhanced SHG with the enhancement factor ranging from 8.3 to 12.5 compared with the Au-Ag NRs.

The prominently enhanced SHG of Au@CdSe heterorods is possibly due to the symmetry breaking of morphology induced by the CdSe overgrowth, as the asymmetric Au@CdSe heterorods may lead to more efficient nonlinear emission [9]. In addition, the plasmonexciton interaction in the Au@CdSe heterorods would lead to more effective linear and nonlinear absorptions [47]. In the metal-semiconductor hybrids, the large local field confinement and enhancement would be produced near the interfacial regions $[48,49]$. All these factors effectively contribute to the nonlinear process of SHG. The $I_{\mathrm{SHG}}(2 \omega)$ excitation spectra of Au@CdSe heterorods with a 

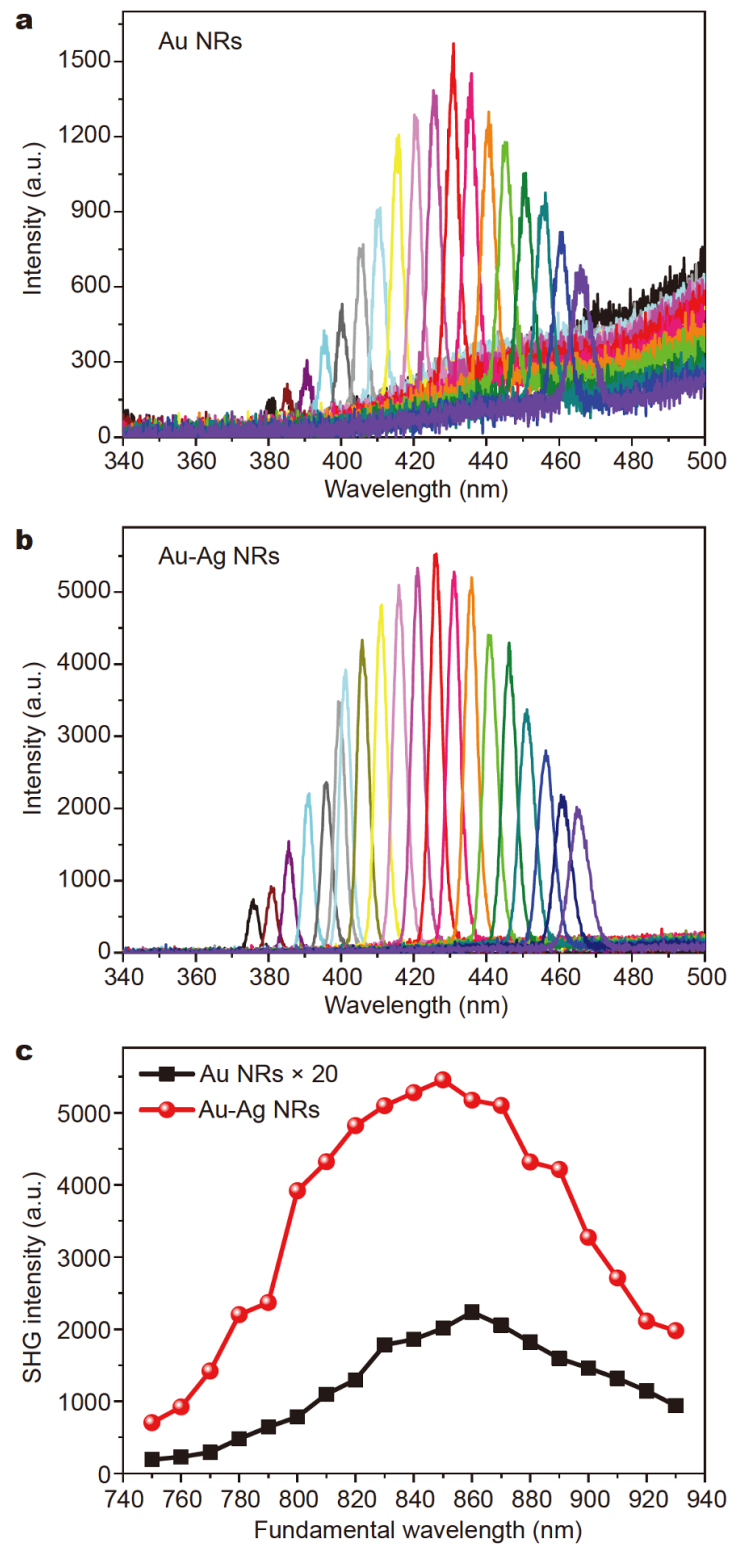

Figure 3 Excitation-wavelength-dependent SHG of Au NRs and Au-Ag NRs. (a, b) SHG emission spectra of Au NRs and Au-Ag NRs as the fundamental wavelength $\lambda_{\mathrm{FW}}$ is varied from 750 to $930 \mathrm{~nm}$. (c) Excitation spectra of $I_{\mathrm{SHG}}(2 \omega)$ for the Au NRs and Au-Ag NRs. The excitation spectrum of Au-Ag NRs shows one peak at $850 \mathrm{~nm}$, while that of Au NRs shows a main peak at $860 \mathrm{~nm}$ and a shoulder peak at $830 \mathrm{~nm}$, respectively.

small amount of CdSe deposition exhibit the single peak line-shape (Fig. S2). It is supposed that the absence of two-peak pattern is attributed to the small $\mu_{\mathrm{Cd}}$ so that there are residual $\mathrm{Ag}$ remaining on the $\mathrm{Au} \mathrm{NRs,} \mathrm{re-}$ straining the efficiency of harmonic resonance energy transfer to the Au interband transition [46]. Meanwhile, the FWHM of excitation spectra is smaller compared with that of Au NRs and Au-Ag NRs, indicating the SHG may
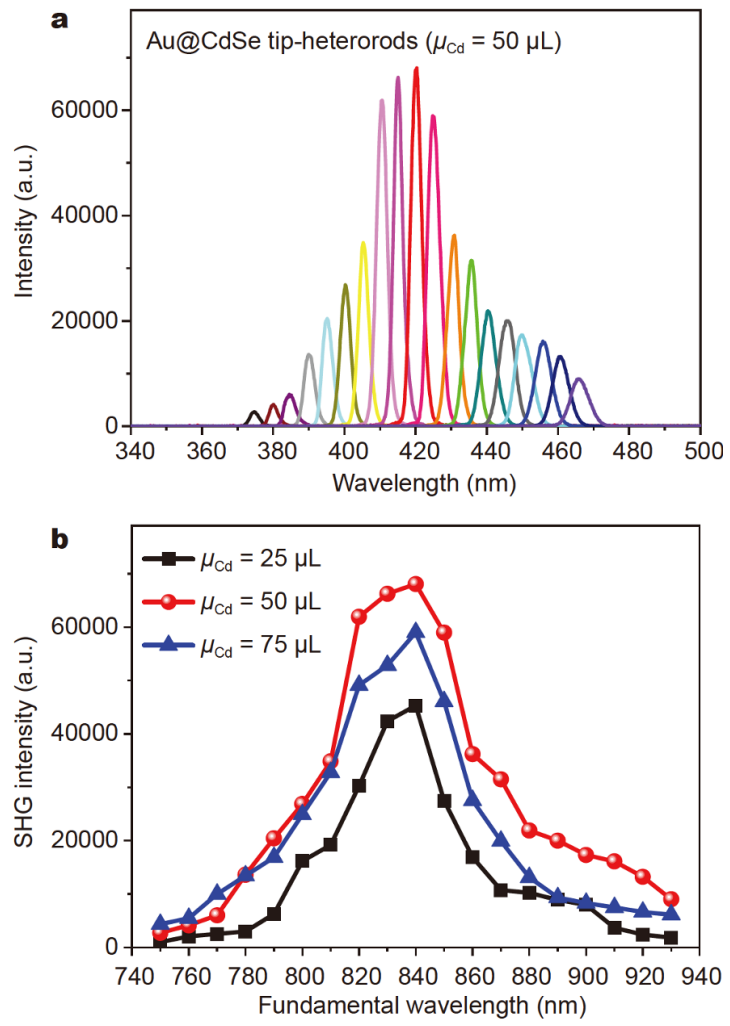

Figure 4 Excitation-wavelength-dependent SHG of Au@CdSe heterorods with a small amount of CdSe deposition. (a) SHG emission spectra of $\mathrm{Au} @ \mathrm{CdSe}$ tip-heterorods with $\mu_{\mathrm{Cd}}=50 \mu \mathrm{L}$ as the fundamental wavelength $\lambda_{\mathrm{FW}}$ is varied from 750 to $930 \mathrm{~nm}$. (b) Excitation spectra of $I_{\mathrm{SHG}}(2 \omega)$ for the Au@CdSe tip-heterorods with $\mu_{\mathrm{Cd}}=25,50$, and $75 \mu \mathrm{L}$, respectively.

be sensitive to the morphology symmetry.

SHG spectra of the asymmetric Au@CdSe side-heterorods When the $\mu_{\mathrm{Cd}}$ is further increased, the Au@CdSe heterorods with a large amount of CdSe deposition are synthesized. The typical SHG emission spectra of the Au@CdSe side-heterorods with $\mu_{\mathrm{Cd}}=200 \mu \mathrm{L}$ are shown in Fig. 5a. The corresponding excitation spectra of $I_{\mathrm{SHG}}(2 \omega)$ for the Au@CdSe heterorods with $\mu_{\mathrm{Cd}}=100,125,200$, and $250 \mu \mathrm{L}$ are presented in Fig. 5b. All the excitation spectra show a two-peak pattern similar with the pure $\mathrm{Au}$ NRs (Fig. S3). In the case with large $\mu_{\mathrm{Cd}}$, the $\mathrm{Ag}$ on the $\mathrm{Au}$ NRs would be run out and the harmonic resonance energy transfer from the $\mathrm{SH}$ excitation to the interband linear excitation of Au may occur again. Compared with the Au@CdSe heterorods with $\mu_{\mathrm{Cd}}=75 \mu \mathrm{L}$, the $I_{\mathrm{SHG}}(2 \omega)$ of the Au@CdSe heterorods with $\mu_{\mathrm{Cd}}=100 \mu \mathrm{L}$ suddenly decreases because of the occurrence of harmonic energy transfer [46]. As the $\mu_{\mathrm{Cd}}$ further increases, the $I_{\mathrm{SHG}}(2 \omega)$ begins to increase. The $I_{\mathrm{SHG}}(2 \omega)$ of the Au@CdSe sideheterorods with $\mu_{\mathrm{Cd}}=200 \mu \mathrm{L}$ show the largest value at 

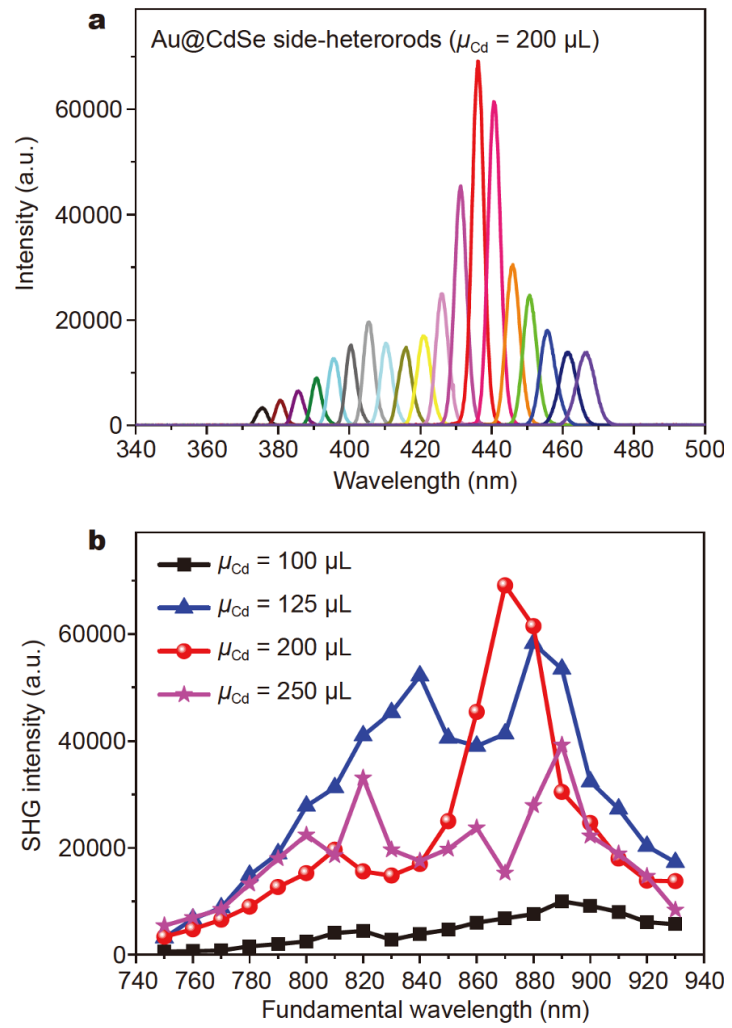

Figure 5 Excitation-wavelength-dependent SHG of Au@CdSe heterorods with a large amount of CdSe deposition. (a) SHG emission spectra of Au@CdSe side-heterorods with $\mu_{\mathrm{Cd}}=200 \mu \mathrm{L}$ as the fundamental wavelength $\lambda_{\mathrm{FW}}$ is varied from 750 to $930 \mathrm{~nm}$. (b) Excitation spectra of $I_{\mathrm{SHG}}(2 \omega)$ for the Au@CdSe heterorods with $\mu_{\mathrm{Cd}}=100,125,200$, and $250 \mu \mathrm{L}$, respectively. All the Au@CdSe heterorods show two extreme peaks in the excitation spectra.

$\lambda_{\mathrm{FW}}=870 \mathrm{~nm}$, which is slightly larger than that of the Au@CdSe tip-heterorods with $\mu_{\mathrm{Cd}}=50 \mu \mathrm{L}$ and 12.7 times that of the Au-Ag NRs. The resonance of $\lambda_{\mathrm{FW}}$ with the LSPR at $862 \mathrm{~nm}$ of the Au@CdSe side-heterorods with $\mu_{\mathrm{Cd}}$ $=200 \mu \mathrm{L}$ is supposed to contribute the optimal $I_{\mathrm{SHG}}(2 \omega)$.

\section{DISCUSSION}

Compared with the "smooth" overgrowth of CdS on the $\mathrm{Au}$ nanoparticles or NRs without etching of $\mathrm{Au}$, the overgrowth of CdSe nanocrystals significantly modifies the shape of the Au NRs owing to the etching effect on the interface during the chemical reaction. The asymmetric overgrowth of CdSe and the etching effect in the Au@CdSe tip- and side-heterorods efficiently break the transverse as well as the longitudinal symmetry of the Au NRs, which significantly improves the out-of-phase of the far-field $\mathrm{SH}$ emission from the opposite sides and ends of the NRs and eventually leads to a very efficient far-field SHG.

The simulations of local field distribution for the $\mathrm{Au}$
NRs and the Au@CdSe heterorods show similar field enhancement (Fig. S4). However, the symmetry of field distribution is broken when the CdSe is grown onto the $\mathrm{Au}$ NRs. Meanwhile, the large local field confinement and enhancement are produced near the interfacial regions. The interaction between plasmonic metals and semiconductors has been found to manipulate the nonlinear responses of the metal-semiconductor hybrids [47-50]. The largely enhanced local field confined at the interfacial regions is expected to enhance the plasmon-exciton interaction such as energy transfer and charge transfer, which could contribute to the SHG emission.

In the excitation spectra of the SHG intensities, the $\mathrm{Au}$ NRs and Au@CdSe side-heterorods both show two extreme peaks in the excitation spectra because of the harmonic energy transfer induced by the local interband transition of Au (see Fig. 2) that is near the SHG emission region (from 375 to $465 \mathrm{~nm}$ ). The $I_{\mathrm{SHG}}(2 \omega)$ of Au@CdSe side-heterorods with $\mu_{\mathrm{Cd}}=200 \mu \mathrm{L}$ is 617.7 -fold larger than that of the bare Au NRs. In contrast, the excitation spectra of Au-Ag NRs and Au@CdSe tip-heterorods show a single peak instead and the $I_{\mathrm{SHG}}(2 \omega)$ of Au@CdSe tipheterorods with $\mu_{\mathrm{Cd}}=50 \mu \mathrm{L}$ is 12.5 -fold larger than that of the Au-Ag NRs. The presence of Ag is supposed to be the reason for restraining the harmonic energy transfer, as the interband energy of $\mathrm{Ag}(3.8 \mathrm{eV})$ does not match the SHG emission wavelength. The underlying physical mechanism of the harmonic energy transfer induced by the local interband transition for the $\mathrm{Au}$ and $\mathrm{Au}-\mathrm{Ag}$ nanocrystals needs to be further investigated. On the other hand, in this work, the amount of deposited $\mathrm{Ag}$ is fixed and the amount of added $\mathrm{Cd}^{2+}$ ions is adjusted to tune the morphology of CdSe. The situation of Ag deposition also influences the morphology of CdSe overgrowth [43]. The influence of initial Ag deposition on the SHG emission may be an interesting topic for future investigation.

The L-SPR of the Au@CdSe heterorods in this study is limited in the range $800-900 \mathrm{~nm}$, then only the fundamental field $\left(\omega_{\mathrm{FW}}\right)$ is resonantly enhanced by the L-SPR of the NRs. The SH field $\left(\omega_{\mathrm{SH}}\right)$ is far off the T-SPR, which indicates that the local and far field of SHG is not enhanced by plasmon resonance. Dual-resonance enhancement effects for both the fundamental and SH fields can be obtained by tuning the size as well as the aspect ratio of the initial Au NRs with appropriate L-SPR and TSPR or using the Ag NRs with T-SPR near $400 \mathrm{~nm}$, which remain to be further studied.

\section{CONCLUSIONS}

In summary, we demonstrate the plasmon-enhanced 
SHG of the asymmetric Au@CdSe heterorods. The $\mathrm{Au} @ \mathrm{CdSe}$ heterorods are tuned from tip- to side-nanostructures by adjusting the concentration of $\mathrm{Cd}^{2+}$ precisely, and the L-SPR significantly red-shifts as the $\mu_{\mathrm{Cd}}$ is increased. Compared with the Au NRs and Au-Ag NRs, the SHG of Au@CdSe heterorods have been greatly enhanced, which is attributed to the asymmetric nanostructures and local field enhancement. The harmonic energy transfer induced by the local interband transition of $\mathrm{Au}$ has also been demonstrated by the excitation-wavelength-dependent SHG in the Au NRs and Au@CdSe side-heterorods. However, for the Au-Ag NRs and $\mathrm{Au} @ \mathrm{CdSe}$ tip-heterorods, the harmonic energy transfer is not observed. The plasmon-enhanced SHG of asymmetric $\mathrm{Au} @ \mathrm{CdSe}$ heterorods enables potential applications in sensing and biological spectroscopy [51-54].

\section{Received 19 December 2019; accepted 2 March 2020; published online 27 March 2020}

1 Kim S, Jin J, Kim YJ, et al. High-harmonic generation by resonant plasmon field enhancement. Nature, 2008, 453: 757-760

2 Ding SJ, Zhang H, Yang DJ, et al. Magnetic plasmon-enhanced second-harmonic generation on colloidal gold nanocups. Nano Lett, 2019, 19: 2005-2011

3 Zengin G, Wersäll M, Nilsson S, et al. Realizing strong light-matter interactions between single-nanoparticle plasmons and molecular excitons at ambient conditions. Phys Rev Lett, 2015, 114: 157401

4 Vasa P, Wang W, Pomraenke R, et al. Real-time observation of ultrafast Rabi oscillations between excitons and plasmons in metal nanostructures with J-aggregates. Nat Photon, 2013, 7: 128-132

5 Li XQ, Yan YJ. Quantum computation with coupled quantum dots embedded in optical microcavities. Phys Rev B, 2002, 65: 205301

6 Stievater TH, Li X, Steel DG, et al. Rabi oscillations of excitons in single quantum dots. Phys Rev Lett, 2001, 87: 133603

7 Zhou L, Swearer DF, Zhang C, et al. Quantifying hot carrier and thermal contributions in plasmonic photocatalysis. Science, 2018, 362: 69-72

8 Swearer DF, Zhao H, Zhou L, et al. Heterometallic antenna-reactor complexes for photocatalysis. Proc Natl Acad Sci USA, 2016, 113: $8916-8920$

9 Ding S, Yang D, Liu X, et al. Asymmetric growth of Au-core/Agshell nanorods with a strong octupolar plasmon resonance and an efficient second-harmonic generation. Nano Res, 2017, 11: 686-695

10 Huang X, El-Sayed IH, Qian W, et al. Cancer cell imaging and photothermal therapy in the near-infrared region by using gold nanorods. J Am Chem Soc, 2006, 128: 2115-2120

11 Anker JN, Hall WP, Lyandres $\mathrm{O}$, et al. Biosensing with plasmonic nanosensors. Nat Mater, 2008, 7: 442-453

12 Kauranen M, Zayats AV. Nonlinear plasmonics. Nat Photon, 2012, 6: $737-748$

13 Wang CY, Chen HY, Sun L, et al. Giant colloidal silver crystals for low-loss linear and nonlinear plasmonics. Nat Commun, 2015, 6: 7734

14 Castro-Lopez M, Brinks D, Sapienza R, et al. Aluminum for nonlinear plasmonics: resonance-driven polarized luminescence of Al, Ag, and Au nanoantennas. Nano Lett, 2011, 11: 4674-4678

15 Rodrigo SG, Harutyunyan H, Novotny L. Coherent control of light scattering from nanostructured materials by second-harmonic generation. Phys Rev Lett, 2013, 110: 177405

16 Czaplicki R, Husu H, Siikanen R, et al. Enhancement of secondharmonic generation from metal nanoparticles by passive elements. Phys Rev Lett, 2013, 110: 093902

17 Aouani H, Rahmani M, Navarro-Cía M, et al. Third-harmonicupconversion enhancement from a single semiconductor nanoparticle coupled to a plasmonic antenna. Nat Nanotech, 2014, 9: 290-294

18 Kim EM, Elovikov SS, Murzina TV, et al. Surface-enhanced optical third-harmonic generation in Ag island films. Phys Rev Lett, 2005, 95: 227402

19 Simkhovich B, Bartal G. Plasmon-enhanced four-wave mixing for superresolution applications. Phys Rev Lett, 2014, 112: 056802

20 Jung $\mathrm{Y}$, Chen $\mathrm{H}$, Tong $\mathrm{L}$, et al. Imaging gold nanorods by plasmonresonance-enhanced four wave mixing. J Phys Chem C, 2009, 113: 2657-2663

21 Kreilkamp LE, Belotelov VI, Chin JY, et al. Waveguide-plasmon polaritons enhance transverse magneto-optical kerr effect. Phys Rev X, 2013, 041019

22 Grunin AA, Zhdanov AG, Ezhov AA, et al. Surface-plasmon-induced enhancement of magneto-optical kerr effect in all-nickel subwavelength nanogratings. Appl Phys Lett, 2010, 97: 261908

23 Farrer RA, Butterfield FL, Chen VW, et al. Highly efficient multiphoton-absorption-induced luminescence from gold nanoparticles. Nano Lett, 2005, 5: 1139-1142

24 Verellen N, Denkova D, Clercq BD, et al. Two-photon luminescence of gold nanorods mediated by higher order plasmon modes. ACS Photonics, 2015, 2: 410-416

25 Boyd RW. Nonlinear Optics. San Diego: Academic Press. 1992

26 Henriques AB, Abramof E, Rappl PHO. Theory of near-gap second harmonic generation in centrosymmetric magnetic semiconductors: europium chalcogenides. Phys Rev B, 2009, 80: 245206

27 Dadap JI, Shan J, Heinz TF. Theory of optical second-harmonic generation from a sphere of centrosymmetric material: smallparticle limit. J Opt Soc Am B, 2004, 21: 1328

28 Bachelier G, Butet J, Russier-Antoine I, et al. Origin of optical second-harmonic generation in spherical gold nanoparticles: local surface and nonlocal bulk contributions. Phys Rev B, 2010, 82: 235403

29 Timbrell D, You JW, Kivshar YS, et al. A comparative analysis of surface and bulk contributions to second-harmonic generation in centrosymmetric nanoparticles. Sci Rep, 2018, 8: 3586

30 Celebrano $\mathrm{M}, \mathrm{Wu} \mathrm{X}$, Baselli $\mathrm{M}$, et al. Mode matching in multiresonant plasmonic nanoantennas for enhanced second harmonic generation. Nat Nanotech, 2015, 10: 412-417

31 Thyagarajan K, Rivier S, Lovera A, et al. Enhanced second-harmonic generation from double resonant plasmonic antennae. Opt Express, 2012, 20: 12860-12865

32 Russier-Antoine I, Benichou E, Bachelier G, et al. Multipolar contributions of the second harmonic generation from silver and gold nanoparticles. J Phys Chem C, 2007, 111: 9044-9048

33 Thyagarajan K, Butet J, Martin OJF. Augmenting second harmonic generation using fano resonances in plasmonic systems. Nano Lett, 2013, 13: 1847-1851

34 Walsh GF, Dal Negro L. Enhanced second harmonic generation by photonic-plasmonic Fano-type coupling in nanoplasmonic arrays. Nano Lett, 2013, 13: 3111-3117

35 Husu H, Siikanen R, Mäkitalo J, et al. Metamaterials with tailored nonlinear optical response. Nano Lett, 2012, 12: 673-677

36 Canfield BK, Husu H, Laukkanen J, et al. Local field asymmetry 
drives second-harmonic generation in noncentrosymmetric nanodimers. Nano Lett, 2007, 7: 1251-1255

37 Linden S, Niesler FBP, Förstner J, et al. Collective effects in secondharmonic generation from split-ring-resonator arrays. Phys Rev Lett, 2012, 109: 015502

38 Valev VK, Smisdom N, Silhanek AV, et al. Plasmonic ratchet wheels: switching circular dichroism by arranging chiral nanostructures. Nano Lett, 2009, 9: 3945-3948

39 Hubert C, Billot L, Adam PM, et al. Role of surface plasmon in second harmonic generation from gold nanorods. Appl Phys Lett, 2007, 90: 181105

40 Rouxel JR, Nguyen TN, Shen H, et al. Enhanced second harmonic generation of gold nanostars: optimizing multipolar radiation to improve nonlinear properties. Opt Express, 2019, 27: 5620-5640

41 Hazra B, Das K, Chandra M. Large second harmonic generation from hollow gold nanoprisms: role of plasmon hybridization and structural effects. Phys Chem Chem Phys, 2017, 19: 18394-18399

42 Liu M, Guyot-Sionnest P. Mechanism of silver(I)-assisted growth of gold nanorods and bipyramids. J Phys Chem B, 2005, 109: 22192-22200

43 Liang S, Liu XL, Yang YZ, et al. Symmetric and asymmetric AuAgCdSe hybrid nanorods. Nano Lett, 2012, 12: 5281-5286

44 Zhao Q, Ji M, Qian H, et al. Controlling structural symmetry of a hybrid nanostructure and its effect on efficient photocatalytic hydrogen evolution. Adv Mater, 2014, 26: 1387-1392

45 Liu XD, Chen K, Ma S, et al. Synthesis of Au/CdSe Janus nanoparticles with efficient charge transfer for improving photocatalytic hydrogen generation. Nanoscale Res Lett, 2019, 14: 349

46 Qiu YH, Ding SJ, Lin YJ, et al. Growth of Au hollow stars and harmonic excitation energy transfer. ACS Nano, 2020, 14: 736-745

47 Nan F, Liang S, Liu XL, et al. Sign-reversed and magnitude-enhanced nonlinear absorption of $\mathrm{Au}-\mathrm{CdS}$ core-shell hetero-nanorods. Appl Phys Lett, 2013, 102: 163112

48 Nan F, Liang S, Wang JH, et al. Tunable plasmon enhancement of gold/semiconductor core/shell hetero-nanorods with site-selectively grown shell. Adv Opt Mater, 2014, 2: 679-686

49 Nan F, Xie FM, Liang S, et al. Growth of metal-semiconductor core-multishell nanorods with optimized field confinement and nonlinear enhancement. Nanoscale, 2016, 8: 11969-11975

50 Shaviv E, Banin U. Synergistic effects on second harmonic generation of hybrid $\mathrm{CdSe}-\mathrm{Au}$ nanoparticles. ACS Nano, 2010, 4: 1529-1538

51 Butet J, Brevet PF, Martin OJF. Optical second harmonic generation in plasmonic nanostructures: from fundamental principles to advanced applications. ACS Nano, 2015, 9: 10545-10562

52 Butet J, Russier-Antoine I, Jonin C, et al. Sensing with multipolar second harmonic generation from spherical metallic nanoparticles. Nano Lett, 2012, 12: 1697-1701

53 Ray PC. Size and shape dependent second order nonlinear optical properties of nanomaterials and their application in biological and chemical sensing. Chem Rev, 2010, 110: 5332-5365

54 Campagnola P. Second harmonic generation imaging microscopy: applications to diseases diagnostics. Anal Chem, 2011, 83: 32243231

Acknowledgements This work was supported by the National Key R\&D Program of China (2017YFA0303402) and the National Natural Science Foundation of China (11874293, 91750113, 11674254 and 11504105). We also appreciate Dr. Zhong-Jian Yang (Central South University) for the simulations.
Author contributions Wu B and Wang PF prepared the samples. Qiu $\mathrm{YH}$ and Wu ZY performed the experiments and analyzed the data. Liang S, Zhou L and Wang QQ guided and supervised the experiments and analysis. All authors contributed to the writing and editing of the manuscript.

Conflict of interest The authors declare that they have no conflict of interest.

Supplementary information Supporting data are available in the online version of the paper.

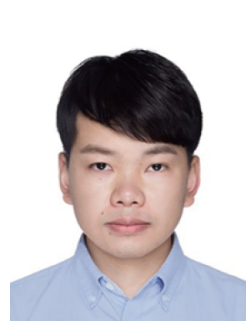

Yun-Hang Qiu received his BSc(Eng) degree from Wuhan University in 2015 and continued to pursue a $\mathrm{PhD}$ degree in physics under the supervision of Prof. Qu-Quan Wang at Wuhan University. His research interests include plasmonics, nonlinear optics and ultrafast dynamics.

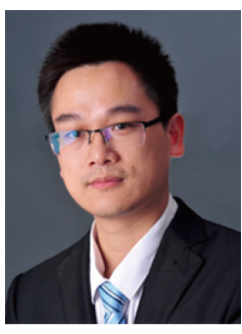

Shan Liang received his MSc and $\mathrm{PhD}$ degrees from Wuhan University. Since 2014, He has been working in the College of Physics and Electronic Science, Hunan Normal University. His research interest includes the building of functionalized hetero nanostructures and devices, as well as their applications in optical, optoelectronic, and nanoenergy fields.

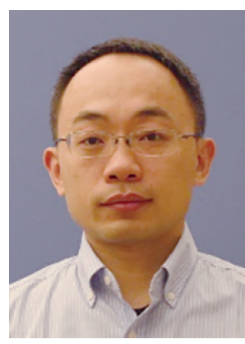

Li Zhou received his BSc, MSc and $\mathrm{PhD}$ degrees from Wuhan University. $\mathrm{He}$ was a visiting scholar at Nanyang Technological University and Georgia Institute of Technology. He is an associate professor at the School of Physics and Technology, Wuhan University. His research interest includes nanophotonics, plasmonics, functional materials and devices at nanoscale as well as their applications in optical, optoelectronic, energy-related, and biomedical fields.

\section{非对称Au@CdSe异质纳米棒的二次谐波增强}

吴彪 ${ }^{1,2 \dagger}$, 王鹏飞 ${ }^{1 \dagger}$, 邱运航 ${ }^{1 \dagger}$, 梁珊 ${ }^{3}$, 武志勇 ${ }^{1}$, 周利 ${ }^{1 *}$, 王取泉 ${ }^{1,4^{*}}$

摘要 本工作对比研究了金纳米棒、金-银纳米棒及非对称 $\mathrm{Au} @ \mathrm{CdSe}$ 异质纳米棒中等离激元增强的二次谐波性质. 通过精确 控制镉离子的浓度, 制备了顶端生长和侧边生长的非对称 $\mathrm{Au} @ \mathrm{CdSe}$ 异质纳米棒, 并实现了等离激元共振波长的调控. 由于非 对称的结构和局域场增强效应, 相比于金纳米棒, 非对称 $\mathrm{Au} @ \mathrm{CdSe}$ 异质纳米棒的二次谐波增强倍数最大可达 617.7. 此外, 二次谐波激 发谱表明在金纳米棒和侧边生长的 $\mathrm{Au} @ \mathrm{CdSe}$ 异质纳米棒中存在着 由金的带间跃迁引起的谐波能量转移. 而由于银的存在, 谐波能量 转移在金-银纳米棒和顶端生长的 $\mathrm{Au} @ \mathrm{CdSe}$ 异质纳米棒中可以忽 略. 非对称 $\mathrm{Au} @ \mathrm{CdSe}$ 异质纳米棒中等离激元增强的二次谐波在传 感、生物光谱等领域都具有潜在应用. 